\title{
Dual Native MS and Cryo-EM Approach to Resolve Heteromeric Protein Assemblies and Subunit Stoichiometry
}

\author{
Irina Novikova ${ }^{1}$, Mowei Zhou ${ }^{1}$, Jared Shaw ${ }^{1}$, Hanjo Hellmann² and James E. Evans ${ }^{1,2, *}$ \\ 1. Environmental Molecular Sciences Laboratory, Richland, WA, USA. \\ 2. School of Biological Sciences, Washington State University, Pullman, WA, USA. \\ * Corresponding author: james.evans@pnnl.gov
}

Vitamin $\mathrm{B}_{6}$ (also called pyridoxine) is a central cofactor for over a hundred enzymatic processes and plays a critical role in the defense mechanism against oxidative stress [1-2]. While plants are able to synthesize vitamin $\mathrm{B}_{6}$ de novo [1,3], humans lack this ability and fully rely on external food nutrition for its intake [4]. In plants, vitamin B6 is synthesized by a PDX1 dodecamer, complexed with a variable number of PDX2 subunits (up to 12). Arabidopsis thaliana has three homologs of PDX1, designated as PDX1.1, PDX1.2 and PDX1.3, along with one homolog of PDX2 [3]. While PDX1.1, PDX1.3 and PDX2 are all active enzymes, PDX1.2 is inactive but still essential for plant development and also the regulation of PDX1.1 and PDX1.3 catalytic activity in vivo [5]. In addition, while the structure of PDX1.3 has been solved by X-ray crystallography [6], the homomeric PDX1.2 pseudoenzyme and PDX1.2/PDX1.3 heterocomplexes remain uncharacterized at the molecular level.

To address these gaps, we combined cell-free expression [7], functional assays, native mass spectrometry, single particle cryo-electron microscopy and other biochemical approaches to evaluate the structure and kinetics of PDX1.2 and its interaction with PDX1.3. Here, we highlight the newly solved structure of homomeric PDX1.2, its assembly into heteromeric complexes with PDX1.3 and propose a new mechanism for how PDX1.2 regulates overall vitamin $\mathrm{B}_{6}$ biosynthesis in the plant. We show that all PDX1.2/PDX1.3 heterocomplexes were found to exist as a population of dodecameric assemblies rather than a single species of one defined stoichiometry. In all co-expression conditions tested, we detected populationa of 6-8 complexes of variable stoichiometry, driven by availability of each protein. A higher ratio of PDX1.2 DNA template fed into the cell-free reaction resulted in a higher number of PDX1.2 subunits present in the heterocomplexes and correlated to a decrease in overall kinetics for PLP synthesis despite improving the stability of the entire complex. Overall, this study highlights a powerful and highly integrated biochemical, mass spectrometry and imaging approach to dissect heteromeric complexes and their association mechanisms [8].

\section{References:}

[1] M Parra, S Stahl and H Hellmann, Cells 7(7) (2018) doi: 10.3390/cells7070084

[2] CC Hsu et al., Food Nutr Res. 59 (2015), p. 25702. doi: 10.3402/fnr.v59.25702

[3] M Tambasco-Studart et al., Proceedings of the National Academy of Sciences of the United States of America 102(38) (2005), p. 13687. doi: 10.1073/pnas.0506228102

[4] MJ Brown and K Beier in "Vitamin B6 Deficiency (Pyridoxine)", (StatPearls, Treasure Island).

[5] JE Leuendorf et al., Mol Plant 3(5) (2010), p. 890. doi: 10.1093/mp/ssq041.

[6] MJ Rodrigues et al., Nat Chem Biol 13(3) (2017), p. 290. doi: 10.1038/nchembio.2273.

[7] IV Novikova et al., Advanced Structural and Chemical Imaging 4 (2018), p. 2198. doi:

10.1186/s40679-018-0062-9 
[8] A portion of this research was supported by NIH grant U24GM129547 and performed at the PNCC at OHSU and accessed through EMSL (grid.436923.9), a DOE Office of Science User Facility sponsored by the Office of Biological and Environmental Research. Additional support was provided by DOE-BER project FWP 66382 and EMSL Strategic Science Area project 50432.
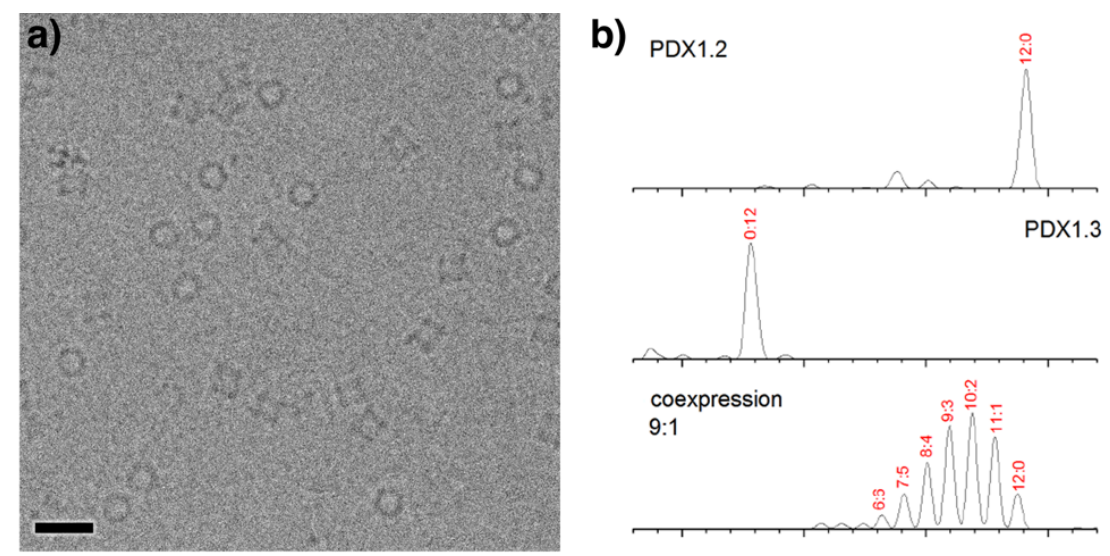

Figure 1. Cryo-EM (a) and Native Mass Spectrometry (b) was performed for homomeric and heteromeric PDX1.2/PDX1.3 complexes to understand the impact of variable stoichiometry of each subunit toward the co-complex structure and function. 